\title{
Influence of Plant Growth Regulators on Seed Yield, Quality and Storability of Soybean [Glycine max (L.) Merrill] Genotypes
}

\author{
Siddaraj*, R.B. Jolli, Jagadish Hosamani and B.N. Motagi \\ Department of Seed Science and Technology, College of Agriculture, Vijayapura, \\ University of Agricultural Sciences, Dharwad, India \\ *Corresponding author
}

\begin{tabular}{|l|}
\hline Ke y w or d s \\
Plant growth \\
regulators, \\
Soybean, \\
Seed yield \\
\hline Article Info \\
\hline Accepted: \\
10 September 2019 \\
Available Online: \\
10 October 2019 \\
\hline
\end{tabular}

\begin{abstract}
A field experiment was conducted during kharif 2018 to study the influence of plant growth regulators on seed yield, quality and storability of soybean genotypes. Among the six genotypes, DSb-21 recorded significantly highest number of branches per plant (4.91) and number of pods per plant (78.34), whereas DSb-31 recorded highest pod length (3.36 $\mathrm{cm})$, number of seeds per pod (3.42) and test weight (14.21 g). Foliar application of cycocel @ 250 ppm furnished significantly higher number of branches per plant (4.46), number of pods per plant $(54.82)$, pod length $(2.67 \mathrm{~cm})$, number of seeds per pod $(2.67)$ and test weight $(13.15 \mathrm{~g})$ as compared to control. Interaction between genotypes and plant growth regulators were found to be non-significant for morpho-physiological and yield attributes of soybean. The genotype Kalitur recorded highest initial seed quality parameters among the genotypes. Initial seed quality parameters viz., seed germination (93.82\%) and seedling vigour index-II (112.60) were found to be significantly higher in salicylic acid @ 50 ppm spray. The interaction effect between genotypes and plant growth regulators were found to be non-significant for seed quality parameters except peroxidase activity and electrical conductivity. Similar trend was noticed up to nine months of storage for seed quality parameters.
\end{abstract}

\section{Introduction}

Soybean [Glycine $\max$ (L.) Merrill] is an annual diploid species grown widely in South East Asia and Europe for its edible oil purpose. India is the fifth largest producer of soybean after USA, China, Brazil and Argentina with an area of 11.66 mha and 8.59 mt production (Anon., 2018). In India, crop has been cultivated largely in states viz., Madhya Pradesh, Maharashtra, Rajasthan,
Andhra Pradesh, Karnataka, Chhattisgarh and Tamil Nadu. Karnataka stands in fifth place cultivated over an area of $2.4 \mathrm{mha}$ and production of $1.4 \mathrm{mt}$ (Anon., 2018).

The yield plateau of soybean is greatly fluctuated by several factors, among them physiological limitations viz., photorespiration, $\mathrm{N}$-fixation metabolism and transpiration leads to lower photosynthetic rate and biomass accumulation. Cycocel is an 
anti-gibberellin compound acting as growth retardant, thereby reduces plant height enhancing lateral growth by checking apical dominance and promoting reproductive growth. Exogenous application of cycocel and salicylic acid through foliar spray are known to increase the seed yield of soybean by modulating the physiological pathways within the plants (Devi et al., 2011; Tarun et al., 2016). Salicylic acid is an orthohydroxybenzoic acid and is a secondary metabolite acting as an analogous of growth regulating substances existing in minor concentration $(1 \mu \mathrm{g} / \mathrm{g})$ in plants such as rice, barley and soybean having diverse role in biotic and abiotic stresses (Raskin et al., 1990; Aydin and Nalbantoglu, 2011).

Soybean is such a crop which loses viability very rapidly under warm and humid tropics (Delouche and Baskin, 1973) leading to poor field emergence and stand establishment. Loss of seed viability in soybean is also correlated with increased rate of lipid peroxidation (Hosamani et al., 2013) in storage due to higher amount of polyunsaturated fatty acids (PUFA) in its seed oil. Hence, in the present study an attempt has been made to know the influence of plant growth regulators on seed yield, quality and storability of soybean genotypes.

\section{Materials and Methods}

A field experiment was conducted at Agricultural Research Station, Arabhavi, Belagavi district, Karnataka, India during kharif 2018 to study the effect of foliar spray of plant growth regulators on seed yield, quality and storability of soybean genotypes. The experiment was laid out in split plot design consisting of different soybean genotypes viz., DSb 21, DSb 23, DSb 31, JS 335, JS 93-05 and Kalitur in main plot and plant growth regulators viz., Control, Cycocel @ 250 ppm and Salicylic acid @ 50 ppm in sub plot. The crop was raised with a spacing of $30 \mathrm{x} 10 \mathrm{~cm}$ by following all the recommended package of practices of University of Agricultural Sciences, Dharwad (Anon., 2016). The plant growth regulators were sprayed at flowering, pod initiation stage and a week prior to harvest. The observations on all the plant morphometric characters were recorded at harvesting stage from a group of five randomly selected plants and average is worked out. The seeds harvested from individual plot were dried to safe moisture level and further used for storage studies over a period of nine months. Harvested seeds were stored in HDPE bags for nine months and observations on per cent seed germination and seedling vigour index-II were recorded at monthly intervals (ISTA, 2018). Electrical conductivity (Agrawal and Dadlani, 1992) of seed leachates was measured at monthly intervals and peroxidase activity (Castillo et al., 1994) was assessed once in three months.

The data collected from field and laboratory experiment was subjected to statistical analysis by using ANOVA test for split plot design and factorial CRD, respectively (Panse and Sukhatme, 1989).

\section{Results and Discussion}

The genotypes differed significantly for morpho-physiological and yield attributing traits of soybean. The genotype DSb 21 recorded significantly highest plant height $(56.06 \mathrm{~cm})$, number of branches per plant (4.91) and number of pods per plant (78.34), whereas DSb 31 recorded statistically highest pod length $(3.36 \mathrm{~cm})$, number of seeds per pod (3.42) and test weight (14.21 g) among the genotypes. This differential genotypic response was due to genetic potential of the genotype and similar differences for morphophysiological and yield attributes were also observed by Kumar et al., (2002) and Chavan (2010) in soybean. Among the plant growth 
regulators, cycocel @ 250 ppm furnished significantly highest number of branches per plant (4.46), number of pods per plant (54.82), pod length $(2.67 \mathrm{~cm})$, number of seeds per pod (2.67), test weight (13.15 g), seed yield per plant (17.27 g) and seed yield (21.21 q/ha) followed by salicylic acid @ 50 ppm (3.82, $50.35,2.40 \mathrm{~cm}, 2.61,12.15 \mathrm{~g}, 15.27 \mathrm{~g}$ and $19.99 \mathrm{q} / \mathrm{ha}$, respectively) as compared to control $(3.43,46.25,2.34 \mathrm{~cm}, 2.39,11.45 \mathrm{~g}$, $12.25 \mathrm{~g}$ and $18.61 \mathrm{q} / \mathrm{ha}$, respectively). Plant height $(42.24 \mathrm{~cm})$ reduced significantly over the control $(47.38 \mathrm{~cm})$ due to foliar spray of cycocel@250 ppm, whereas salicylic acid @ 50 ppm promoted plant height $(53.0 \mathrm{~cm})$. Cycocel is a growth retardant which checks apical dominance and acts as an antigibberellin compound inhibiting biosynthesis of $\mathrm{GA}_{3}$ in plants. Hence, foliar spray of cycocel resulted in reduced plant height. Foliar application of plant growth regulators strengthened physiological relationship between source and sink resulting in effective partitioning and translocation of photosynthates from leaves to seeds within the plant. Similar results for yield attributes of soybean as influenced by cycocel and salicylic acid spray were also reported by Kalyankar et al., (2008), Devi et al., (2011), Tarun et al., (2016) and Solanke et al., (2018) in soybean and Kumar et al., (2018) with salicylic acid spray in green gram.

The interaction effects between genotypes and plant growth regulators were found to be nonsignificant for morpho-physiological and yield attributes of soybean. Among the different treatment combinations, DSb 21 with foliar spray of cycocel @ 250 ppm $\left(\mathrm{G}_{1} \mathrm{P}_{2}\right)$ recorded significantly highest number of branches per plant (5.60), number of pods per plant (84.03), seed yield per plant (29.14 g) and seed yield $(28.56 \mathrm{q} / \mathrm{ha})$, whereas DSb 31 with foliar application of cycocel @ 250 ppm $\left(\mathrm{G}_{3} \mathrm{P}_{2}\right)$ recorded significantly highest pod length (3.77 $\mathrm{cm}$ ), number of seeds per pod (3.73) and test weight (15.21 g). Similar non-significant interaction variations for morphophysiological and yield attributes were also reported by Devi et al., (2011) and Kaur et al., (2015) in soybean with cycocel and salicylic acid foliar spray.

Among the genotypes, Kalitur recorded highest initial seed quality parameters viz., seed germination $(96.31 \%)$, seedling vigour index-II (123.06) and peroxidase activity (37.39 $\mu$ moles $\mathrm{H}_{2} \mathrm{O}_{2} / \mathrm{cm} / \mathrm{min} / \mathrm{g}$ ), whereas electrical conductivity was found to be significantly lowest $(0.035 \mathrm{dS} / \mathrm{m})$. Such genotypic variability for seed quality parameters were also observed by Vanangamudi (1988), Hosamani et al., (2013) and Yaklich et al., (1979) in soybean. Foliar spray of salicylic acid @ 50 ppm recorded significantly higher initial seed quality parameters viz., seed germination (93.82\%), seedling vigour index-II (112.60) and peroxidase activity $(19.57 \mu$ moles $\mathrm{H}_{2} \mathrm{O}_{2} / \mathrm{cm} / \mathrm{min} / \mathrm{g}$ ), whereas electrical conductivity was found to be significantly lowest $(0.048 \mathrm{dS} / \mathrm{m})$ followed by cycocel @ $250 \mathrm{ppm}(91.77 \%, 108.31,16.90 \mu$ moles $\mathrm{H}_{2} \mathrm{O}_{2} / \mathrm{cm} / \mathrm{min} / \mathrm{g}$ and $0.061 \mathrm{dS} / \mathrm{m}$, respectively) as compared to control (89.95\%, 103.29, $15.27 \mu$ moles $\mathrm{H}_{2} \mathrm{O}_{2} / \mathrm{cm} / \mathrm{min} / \mathrm{g}$ and 0.074 $\mathrm{dS} / \mathrm{m}$, respectively). Similar results were also drawn by Kuchlan et al., (2017) in soybean genotype JS 335 with foliar spray of salicylic acid. Seed germination and seedling vigour index-II enhanced significantly due to increased seed vigour potential as a result of foliar spray of plant growth regulators. Nambiar et al., (2006) reported similar kind of results with foliar application of plant growth regulators on seed quality and storability of soybean in JS 335 cultivar over a period of six months of storage. The interaction effects between genotypes and plant growth regulators were found to be significant for electrical conductivity and peroxidase activity (Table 1 and 2). 
Table.1 Effect of foliar spray of plant growth regulators on morpho-physiological and yield attributing traits of soybean genotypes

\begin{tabular}{|c|c|c|c|c|c|c|c|c|}
\hline Treatments & $\begin{array}{c}\text { Plant } \\
\text { height at } \\
\text { harvest } \\
(\mathrm{cm})\end{array}$ & $\begin{array}{c}\text { Number } \\
\text { of } \\
\text { branches } \\
\text { per plant } \\
\text { at harvest }\end{array}$ & $\begin{array}{c}\text { Number } \\
\text { of pods } \\
\text { per plant }\end{array}$ & $\begin{array}{c}\text { Pod } \\
\text { length } \\
(\mathrm{cm})\end{array}$ & $\begin{array}{l}\text { Number } \\
\text { of seeds } \\
\text { per pod }\end{array}$ & $\begin{array}{c}\text { Test } \\
\text { weight (g) }\end{array}$ & $\begin{array}{l}\text { Seed yield } \\
\text { per plant } \\
\text { (g) }\end{array}$ & $\begin{array}{l}\text { Seed yield } \\
(\mathbf{q} / \mathbf{h a})\end{array}$ \\
\hline \multicolumn{9}{|l|}{ Genotypes: (G) } \\
\hline $\mathrm{G}_{1}: \mathrm{DSb}-21$ & 56.06 & 4.91 & 78.34 & 2.68 & 2.67 & 13.43 & 25.73 & 27.95 \\
\hline$G_{2}:$ DSb-23 & 51.40 & 4.71 & 68.33 & 2.66 & 2.42 & 12.92 & 17.30 & 26.17 \\
\hline$G_{3}: D S b-31$ & 46.63 & 3.33 & 34.40 & 3.36 & 3.42 & 14.21 & 14.21 & 24.02 \\
\hline$G_{4}:$ JS 335 & 50.69 & 3.93 & 51.33 & 2.41 & 2.39 & 12.21 & 15.30 & 18.87 \\
\hline Gs: $_{5}$ JS 9305 & 50.30 & 3.72 & 42.30 & 2.16 & 2.30 & 11.47 & 11.92 & 15.30 \\
\hline G $_{6}$ : Kalitur & 30.15 & 2.80 & 28.12 & 1.57 & 2.13 & 9.26 & 5.12 & 7.30 \\
\hline S.Em \pm & 1.67 & 0.20 & 1.99 & 0.08 & 0.10 & 0.49 & 0.60 & 64.85 \\
\hline CD at $5 \%$ & 5.25 & 0.63 & 6.26 & 0.26 & 0.31 & 1.55 & 1.90 & 204.36 \\
\hline \multicolumn{9}{|c|}{ Plant growth regulators: $(\mathbf{P})$} \\
\hline$P_{1}$ : Control & 47.38 & 3.43 & 46.25 & 2.34 & 2.39 & 11.45 & 12.25 & 18.61 \\
\hline$P_{2}:$ Cycocel @ 250 ppm & 42.24 & 4.46 & 54.82 & 2.67 & 2.67 & 13.15 & 17.27 & 21.21 \\
\hline $\begin{array}{l}\mathbf{P}_{3}: \text { Salicylic acid @ } 50 \\
\text { ppm }\end{array}$ & 53.00 & 3.82 & 50.35 & 2.40 & 2.61 & 12.15 & 15.27 & 19.99 \\
\hline S.Em \pm & 0.82 & 0.08 & 0.75 & 0.04 & 0.04 & 0.20 & 0.31 & 20.26 \\
\hline CD at $5 \%$ & 2.40 & 0.25 & 2.18 & 0.11 & 0.12 & 0.59 & 0.90 & 59.14 \\
\hline \multicolumn{9}{|l|}{ Interactions: (G X P) } \\
\hline $\mathbf{G}_{1} \mathbf{P}_{1}$ & 54.14 & 4.20 & 72.00 & 2.58 & 2.56 & 12.48 & 21.69 & 27.22 \\
\hline $\mathbf{G}_{1} \mathbf{P}_{2}$ & 53.14 & 5.60 & 84.03 & 2.85 & 2.72 & 14.67 & 29.14 & 28.56 \\
\hline $\mathbf{G}_{1} \mathbf{P}_{3}$ & 60.90 & 4.93 & 79.00 & 2.61 & 2.71 & 13.13 & 26.35 & 28.08 \\
\hline $\mathbf{G}_{2} \mathbf{P}_{1}$ & 52.60 & 4.05 & 66.01 & 2.57 & 2.15 & 12.17 & 14.31 & 24.99 \\
\hline $\mathbf{G}_{2} \mathbf{P}_{2}$ & 45.87 & 5.33 & 70.83 & 2.82 & 2.58 & 13.93 & 19.71 & 27.23 \\
\hline $\mathbf{G}_{2} \mathbf{P}_{3}$ & 55.72 & 4.73 & 68.13 & 2.61 & 2.51 & 12.66 & 17.90 & 26.27 \\
\hline $\mathbf{G}_{3} \mathbf{P}_{1}$ & 47.15 & 3.20 & 31.52 & 3.02 & 2.99 & 13.52 & 13.23 & 22.45 \\
\hline $\mathbf{G}_{3} \mathbf{P}_{2}$ & 39.87 & 3.50 & 37.54 & 3.77 & 3.73 & 15.21 & 15.10 & 26.08 \\
\hline $\mathbf{G}_{3} \mathbf{P}_{3}$ & 52.87 & 3.30 & 34.15 & 3.28 & 3.53 & 13.91 & 14.29 & 23.53 \\
\hline $\mathbf{G}_{\mathbf{4}} \mathbf{P}_{1}$ & 50.66 & 3.47 & 47.56 & 2.32 & 2.26 & 11.45 & 11.58 & 17.32 \\
\hline $\mathbf{G}_{4} \mathbf{P}_{2}$ & 44.46 & 4.60 & 56.41 & 2.57 & 2.48 & 12.61 & 18.83 & 20.67 \\
\hline $\mathbf{G}_{4} \mathbf{P}_{3}$ & 56.97 & 3.73 & 50.01 & 2.33 & 2.43 & 12.58 & 15.49 & 18.63 \\
\hline $\mathbf{G}_{5} \mathbf{P}_{1}$ & 51.08 & 3.00 & 36.27 & 2.08 & 2.29 & 10.54 & 9.48 & 13.06 \\
\hline $\mathbf{G}_{5} \mathbf{P}_{2}$ & 44.28 & 4.70 & 48.31 & 2.25 & 2.29 & 12.42 & 13.92 & 16.93 \\
\hline $\mathbf{G}_{5} \mathbf{P}_{3}$ & 55.55 & 3.47 & 42.33 & 2.14 & 2.31 & 11.47 & 12.35 & 15.92 \\
\hline $\mathbf{G}_{6} P_{1}$ & 28.65 & 2.63 & 24.13 & 1.50 & 2.07 & 8.56 & 3.18 & 6.61 \\
\hline $\mathbf{G}_{6} \mathbf{P}_{2}$ & 25.80 & 3.03 & 31.79 & 1.77 & 2.19 & 10.08 & 6.92 & 7.76 \\
\hline $\mathbf{G}_{6} \mathbf{P}_{3}$ & 36.00 & 2.73 & 28.45 & 1.45 & 2.14 & 9.14 & 5.24 & 7.53 \\
\hline S.Em \pm & 2.02 & 0.21 & 1.83 & 0.09 & 0.10 & 0.50 & 0.75 & 49.63 \\
\hline CD at $5 \%$ & NS & NS & NS & NS & NS & NS & NS & NS \\
\hline
\end{tabular}

*NS-Non-significant 
Table.2 Effect of foliar spray of plant growth regulators on seed quality and storability of soybean genotypes

\begin{tabular}{|c|c|c|c|c|c|c|c|c|}
\hline \multirow[t]{2}{*}{ Treatments } & \multicolumn{2}{|c|}{$\begin{array}{c}\text { Seed germination } \\
(\%)\end{array}$} & \multicolumn{2}{|c|}{$\begin{array}{l}\text { Seedling vigour } \\
\text { index-II }\end{array}$} & \multicolumn{2}{|c|}{$\begin{array}{c}\text { Electrical } \\
\text { conductivity }(\mathrm{dS} / \mathrm{m})\end{array}$} & \multicolumn{2}{|c|}{$\begin{array}{c}\text { Peroxidase } \\
\text { activity } \\
(\mu \text { moles } \\
\left.\mathrm{H}_{2} \mathrm{O}_{2} / \mathrm{cm} / \mathrm{min} / \mathrm{g}\right)\end{array}$} \\
\hline & Initial & 9 MAS & Initial & 9 MAS & Initial & 9 MAS & Initial & 9 MAS \\
\hline \multicolumn{9}{|l|}{ Genotypes (G): } \\
\hline$G_{1}: D S b-21$ & 95.35 (77.76) & $79.22(62.88)$ & 118.02 & 58.95 & 0.049 & 0.466 & 26.02 & 67.41 \\
\hline$G_{2}: D S b-23$ & $92.51(74.31)$ & 77.93 (61.99) & 108.98 & 56.83 & 0.056 & 0.475 & 13.84 & 60.01 \\
\hline$G_{3}: D S b-31$ & $89.38(70.98)$ & $75.01(60.02)$ & 102.57 & 45.86 & 0.059 & 0.489 & 9.99 & 52.94 \\
\hline$G_{4}:$ JS 335 & $88.32(70.08)$ & $71.04(57.45)$ & 95.31 & 41.59 & 0.093 & 0.537 & 6.33 & 30.38 \\
\hline G $_{5}:$ JS 9305 & 89.19 (71.13) & $72.81(58.58)$ & 100.46 & 47.21 & 0.073 & 0.508 & 9.90 & 46.73 \\
\hline G $_{6}$ : Kalitur & $96.31(79.21)$ & 80.95 (64.13) & 123.06 & 67.93 & 0.035 & 0.372 & 37.39 & 73.81 \\
\hline S.Em \pm & 0.685 & 0.11 & 0.71 & 0.44 & 0.001 & 0.007 & 0.21 & 0.54 \\
\hline CD at $1 \%$ & 2.633 & 0.43 & 2.73 & 1.71 & 0.004 & 0.029 & 0.81 & 2.09 \\
\hline \multicolumn{9}{|l|}{ Plant growth regulators $(\mathbf{P})$ : } \\
\hline$P_{1}$ : Control & $89.95(71.85)$ & $74.56(59.77)$ & 103.29 & 49.40 & 0.074 & 0.545 & 15.27 & 51.67 \\
\hline$P_{2}:$ Cycocel @ 250 ppm & $91.77(73.78)$ & $76.27(60.91)$ & 108.31 & 53.48 & 0.061 & 0.463 & 16.90 & 55.61 \\
\hline$P_{3}:$ Salicylic acid @ 50 ppm & $93.82(76.10)$ & $77.65(61.85)$ & 112.60 & 56.31 & 0.048 & 0.415 & 19.57 & 58.36 \\
\hline S.Em \pm & 0.484 & 0.08 & 0.50 & 0.31 & 0.001 & 0.005 & 0.15 & 0.38 \\
\hline CD at $1 \%$ & 1.862 & $\mathbf{0 . 3 0}$ & 1.93 & 1.21 & 0.003 & $\mathbf{0 . 0 2 0}$ & 0.57 & 1.48 \\
\hline \multicolumn{9}{|l|}{ Interactions (G X P): } \\
\hline $\mathbf{G}_{1} \mathbf{P}_{1}$ & $94.16(76.13)$ & $78.27(62.22)$ & 114.37 & 56.67 & 0.057 & 0.511 & 24.85 & 62.31 \\
\hline $\mathbf{G}_{1} \mathbf{P}_{2}$ & $95.49(77.87)$ & $79.11(62.81)$ & 118.09 & 58.89 & 0.048 & 0.471 & 26.23 & 67.50 \\
\hline $\mathbf{G}_{\mathbf{1}} \mathbf{P}_{3}$ & $96.40(79.27)$ & $80.27(63.63)$ & 121.59 & 61.28 & 0.041 & 0.417 & 26.97 & 72.42 \\
\hline $\mathbf{G}_{2} \mathbf{P}_{1}$ & $90.74(72.43)$ & $76.18(60.79)$ & 104.85 & 53.79 & 0.066 & 0.533 & 11.74 & 55.23 \\
\hline $\mathbf{G}_{2} \mathbf{P}_{2}$ & $92.23(73.91)$ & $78.24(62.20)$ & 108.92 & 56.88 & 0.056 & 0.459 & 12.03 & 60.61 \\
\hline $\mathbf{G}_{2} \mathbf{P}_{3}$ & $94.56(76.59)$ & 79.37 (62.98) & 113.18 & 59.81 & 0.047 & 0.433 & 17.76 & 64.18 \\
\hline $\mathbf{G}_{3} \mathbf{P}_{1}$ & $86.80(68.72)$ & $72.60(58.44)$ & 95.88 & 40.77 & 0.065 & 0.563 & 9.02 & 48.74 \\
\hline $\mathbf{G}_{3} \mathbf{P}_{2}$ & $88.57(70.27)$ & $75.14(60.09)$ & 102.78 & 46.76 & 0.061 & 0.483 & 9.13 & 53.37 \\
\hline $\mathbf{G}_{3} \mathbf{P}_{3}$ & $92.21(73.95)$ & $77.30(61.54)$ & 109.04 & 50.04 & 0.050 & 0.421 & 11.82 & 56.72 \\
\hline $\mathbf{G}_{4} \mathbf{P}_{1}$ & $86.39(68.36)$ & $69.64(56.57)$ & 91.21 & 38.01 & 0.123 & 0.646 & 5.18 & 26.62 \\
\hline $\mathbf{G}_{4} \mathbf{P}_{2}$ & $88.08(69.81)$ & $70.86(57.33)$ & 95.04 & 41.36 & 0.090 & 0.518 & 5.85 & 30.30 \\
\hline $\mathbf{G}_{4} \mathbf{P}_{3}$ & $90.50(72.07)$ & $72.60(58.44)$ & 99.69 & 45.40 & 0.065 & 0.447 & 7.96 & 34.21 \\
\hline $\mathbf{G}_{5} \mathbf{P}_{1}$ & $87.08(68.98)$ & $71.25(57.57)$ & 94.71 & 43.42 & 0.088 & 0.586 & 9.00 & 45.22 \\
\hline $\mathbf{G}_{5} \mathbf{P}_{2}$ & $89.56(71.24)$ & $73.04(58.72)$ & 101.18 & 47.65 & 0.071 & 0.501 & 9.92 & 47.41 \\
\hline $\mathbf{G}_{5} \mathbf{P}_{3}$ & $91.50(73.16)$ & $74.16(59.45)$ & 105.50 & 50.58 & 0.061 & 0.437 & 10.78 & 47.54 \\
\hline $\mathbf{G}_{6} P_{1}$ & $94.53(76.50)$ & $79.44(63.04)$ & 118.70 & 63.73 & 0.042 & 0.433 & 31.86 & 71.88 \\
\hline $\mathbf{G}_{6} \mathbf{P}_{2}$ & $96.66(79.58)$ & $81.21(64.31)$ & 123.85 & 69.33 & 0.036 & 0.348 & 38.22 & 74.49 \\
\hline $\mathbf{G}_{6} \mathbf{P}_{3}$ & $97.73(81.54)$ & $82.19(65.04)$ & 126.62 & 70.74 & 0.026 & 0.334 & 42.10 & 75.07 \\
\hline S.Em \pm & 1.186 & 0.19 & 1.23 & 0.77 & 0.002 & 0.01 & 0.37 & 0.94 \\
\hline CD at $1 \%$ & NS & NS & NS & NS & 0.006 & 0.05 & 1.40 & 3.62 \\
\hline
\end{tabular}

* NS-Non-significant, MAS-Months after storage

* Values in parenthesis are arcsine transformed germination values 
The increased peroxidase activity as a result of foliar spray of salicylic acid @ 50 ppm may be due to up-regulation of genes involved in biosynthesis of antioxidant enzymes (Mohamed et al., 2017) alleviating catalytic activity of peroxidase. Electrical conductivity of seed leachates reduced significantly as a result of increased peroxidase activity which scavenges free radicals and organic peroxides in the cell. Thus, preventing oxidative damage to cell membrane thereby reducing release of volatile aldehydes and electrolytes from the seed. Jakhar and Sheokand (2015) reported that foliar application of salicylic acid modulates oxidative stress in soybean plant and reduces rate of lipid peroxidation process by alleviating activity of antioxidant enzymes. Similar trend was noticed for seed germination, seedling vigour index-II, electrical conductivity and peroxidase activity of soybean genotypes up to nine months of storage.

\section{References}

Agrawal, P. K. and Dadlani, M. (1992). Techniques in Seed Science and Technology. South Asian Publisher, New Delhi 114-120.

ISTA (2018). International Rules for Seed Testing. Seed. Sci. Tech., 44(2): 27-32.

Anonymous, 2016, Package of practices for agricultural crops, University of Agricultural Sciences, Dharwad, pp.169-178.

Anonymous, 2018, Agricultural Statistics at a glance. Agricultural statistics division, Directorate of Economics and Statistics, Department of Agriculture, Cooperation and Farmers Welfare, Ministry of agriculture and Farmers Welfare, Government of India, New Delhi.

Aydin, B. and Nalbantoglu, B. (2011). Effects of cold and salicylic acid treatments on nitrate reductase activity in spinach leaves. Turkish J. Biol., 35: 443-448.

Chavan, N. G. (2010). Characterization of soybean [Glycine max (L.) Merrill] genotypes through morphological, chemical, molecular markers and image analyzer. M.Sc. (Agri.) Thesis, Univ. Agric. Sci., Dharwad, Karnataka (India).

Delouche, J. C. and Baskin, C. C. (1973). Accelerated ageing techniques for predicting the relative storability of seed lots. Seed. Sci. Tech., 1: 427-452.

Devi, K. N., Vyas, A. K., Singh, M. S. and Singh, N. G. (2011). Effect of bioregulators on growth, yield and chemical constituents of soybean (Glycine max L.). J. Agric. Sci., 3(4): 151-159.

Hosamani, J., Dadlani, M., Santha, I. M., Kumar, M. A. and Jacob, S. R. (2013). Biochemical phenotyping of soybean (Glycine $\max$ L.) genotypes to establish the role of lipid peroxidation and antioxidant enzymes in seed longevity. Agric. Res., 2(2): 119-126.

Jakhar, S. and Sheokand, M. (2015). Effect of foliar application of salicylic acid on photosynthetic pigments and antioxidative enzymes of soybean plant. Int. J. Appl. Pure Sci. Agric., 1: 715.

Kalyankar, S. V., Kadam, G. R., Borgaonkar, S. B., Deshmukh, D. D. and Kadam, B. P. (2008). Effect of foliar application of growth regulators on seed yield and yield components of soybean [Glycine $\max$ (L.) Merrill]. Asian J. Bio. Sci., 3(1): 229-230.

Kaur, J., Ram, H., Gill, B. S. and Kaur, J. (2015). Agronomic performance and economic analysis of soybean (Glycine $\max$ L.) in relation to growth regulating substances in Punjab, India. Leg. Res., 38: 603-608.

Kuchlan, P., Kuchlan, M. K. and Husain, S. M. (2017). Effect of foliar application 
of growth activator, promoter and antioxidant on seed quality of soybean. Leg. Res., 40 (2): 313-318.

Kumar, P., Hiremath, S. M., Deshmukh, P. S. and Kushwaha, S. R. (2002). Effect of growth regulators on growth, yield and metabolism in soybean genotypes. Indian J. Agri. Res., 36(4): 254-258.

Kumar, R., Yadav, R. K., Sharma, N. and Nehal, N. (2018). Influence of plant growth regulators on yield and yield attributes of green gram (Vigna radiata L.). J. Pharmacognosy \& Phytochemistry.2: 98-100.

Mohamed, A. El-Esawi., Hosam, O. Elansary., Nader, A. El-Shanhorey., Amal, M. E. Abdel-Hamid., Hayassam, M. Ali and Mohamed, S. Elshikh. (2017). Salicylic acid-regulated antioxidant mechanisms and gene expression enhance Rosemary performance under saline conditions, Front physiol., 8: 716.

Nambiar, P. K. M., Bharud, R. W., Manjare, M. R., Dumbre, A. D. and Khan, B. H. (2006). Effect of pre-harvest applications of plant growth regulators on the seed quality and storability of soybean [Glycine $\max$ (L.) Merrill]. M.Sc. (Agri.) Thesis, Mahatma Phule Krishi Vidyapeeth, Maharashtra (India). Panse, V. G. and Sukhatme, P. V. (1989).
Statistical methods for agricultural workers. $4^{\text {th }}$ Edn, ICAR Publications, New Delhi.

Raskin, I., Skubatz, H., Tang, W. and Meeuse, B. J. D. (1990). Salicylic acid levels in thermogenic and nonthermogenic plants. Ann. Bot., 66: 376378.

Solanke, A. P., Pawar, G. S., Dhage, R. S. and Kamble, B. G. (2018). Effect of plant growth regulators on growth and yield of soybean (Glycine max L.) applied at different stages. Int. J. Chem. Stud., 6(5): 2962-2966.

Tarun, K., Samaiya, R. K., Yogendra, S., Dwivedi, S. K. and Meena, K. C. (2016). Effect of foliar application of plant growth retardants on growth, yield and yield attributing parameters of soybean (Glycine max L.). Int. J. Agric. Sci., 8(50): 2158-2162.

Vanangamudi, K. (1988). Storability of soybean seed as influenced by the variety, seed size and storage container. Seed Res., 16(1): 81-87.

Yaklich, R.W., Kulik, M. M. and Garrison, C. S. (1979). Evaluation of vigor in soybean seeds: Influences of date of planting and soil type on emergence stand and yield. Crop Sci., 19(2): 242246.

\section{How to cite this article:}

Siddaraj, R.B. Jolli, Jagadish Hosamani and Motagi, B.N. 2019. Influence of Plant Growth Regulators on Seed Yield, Quality and Storability of Soybean [Glycine max (L.) Merrill] Genotypes. Int.J.Curr.Microbiol.App.Sci. 8(10): 1166-1172.

doi: https://doi.org/10.20546/ijcmas.2019.810.136 